\title{
Saúde Mental Infanto-juvenil: a necessidade de Políticas Públicas mais efetivas pelo Estado.
}

\author{
Sidney Medeiros de Oliveira ${ }^{1}$; Messias Santos da Silva ${ }^{2}$; Michelle Carolina Alves Nogueira ${ }^{3}$
}

\begin{abstract}
Resumo: O estudo das enfermidades mentais infanto-juvenis é de grande valia, tendo em vista que o conhecimento e divulgação das características presentes nas doenças mentais em crianças, permite o reconhecimento das debilidades cerebrais com mais facilidade, com o escopo de tomar as medidas cabíveis a sua remediação o mais breve possível. O objetivo deste estudo é discutir sobre a necessidade de políticas públicas mais efetivas pelo Estado para a Saúde mental infanto-juvenil. Os resultados demonstraram que ainda há carência de políticas de saúde mental específicas, mais direcionadas ao público infantil. Estes acusam uma urgência de ações mais efetivas, além de estudos que possam fundamentar a elaboração de ações políticas adequadas.
\end{abstract}

Palavras-chave: Saúde mental. Políticas públicas. Enfermidades infanto-juvenis.

\section{Child-juvenile Mental Health: the need for more effective Public Policies by the State.}

\begin{abstract}
The study of child-juvenile mental illnesses is of great value, since knowledge and dissemination of the characteristics present in mental illness in children allows the recognition of cerebral weaknesses more easily, with the scope of taking the appropriate measures remedy as soon as possible. The objective of this study is to discuss the need for more effective public policies by the State for children's mental health. The results showed that there is still a shortage of specific mental health policies aimed at children. These accuse an urgency of more effective actions, in addition to studies that can support the development of appropriate political actions.
\end{abstract}

Keywords: Mental health. Public policy. Child-juvenile diseases.

\section{Introdução}

No sistema de saúde atualmente vigente no Brasil, é notória a desproporção entre a necessidade e a oferta de atenção em saúde mental específica para o público infanto-juvenil. Essa desproporção está presente em todos os países, independente da condição financeira e cultura local, sendo mais evidente nos países subdesenvolvidos.

\footnotetext{
${ }^{1}$ Educador Físico pela Universidade Federal de Pernambuco. Doutorando em Ciências da saúde pela Faculdade de Medicina do ABC, São Paulo, Brasil. Contato: sidneymeolli@gmail.com;

${ }^{2}$ Acadêmico de Pedagogia pela Faculdade da Cidade de Escada - FAESC, Pernambuco, Brasil. Contato: msantos.sociedadejusta@hotmail.com;

${ }^{3}$ Graduanda em Direito pela Faculdade Metropolitana da Grande Recife - Pernambuco, Brasil. Contato: michellenogueira2@hotmail.com.
}

748 Id on Line Rev. Mult. Psic. V.12, N. 41, p. 748-759, 2018 - ISSN 1981-1179 Edição eletrônica em http://idonline.emnuvens.com.br/id 
O estudo das enfermidades mentais infanto-juvenis é de grande valia, tendo em vista que o conhecimento e divulgação das características presentes nas doenças mentais em crianças, permite o reconhecimento das debilidades cerebrais com mais facilidade, com o escopo de tomar as medidas cabíveis a sua remediação o mais breve possível.

É importante investir no estudo, bem como aprimoração do setor da saúde, em relação as enfermidades mentais que possam acometer crianças e adolescentes, é visível na Política Nacional de Saúde Mental, que definiu diretrizes sobre o tema, dentre essas destacam-se: (i) a implementação de serviços de saúde mental voltados para o público infanto-juvenil, pelo SUS, com os Centros de Atenção Psicossocial Infanto-Juvenil (CAPSi); e (ii) o desenvolvimento de estratégias que visem a articulação do setor de saúde mental com os demais relacionados com a assistência infanto-juvenil, com vistas a conferir uma atenção completa aos menores.

Antigamente, os estudos envolvendo enfermidades mentais não eram muito valorizados, e os pacientes eram simplesmente trancados em hospitais psiquiátricos e, por vezes, esquecidos no local pelo resto de suas vidas. Isso não apenas revela uma ineficiência do tratamento, como também revela ser uma forma de exclusão social dos enfermos, que eram tratados friamente como loucos.

Tendo em vistas a situação desumana as quais os doentes mentais eram submetidos com vistas ao seu tratamento, diversos atores sociais lutaram em prol de uma Reforma Psiquiátrica no país, que foi efetivada em 2001, com a criação da Lei no 10.216 (Lei de Saúde Mental). Assim, resta reconhecido que a saúde mental dos menores se trata de questão de saúde pública, devendo, dessa forma, ser disponibilizada por meio do Sistema Único de Saúde (SUS).

Esse movimento reformista foi decisivo na mudança das práticas no sistema de saúde direcionadas aos portadores de transtornos psíquicos, que agora passam a incentivar o desenvolvimento de políticas com o escopo de diminuir leitos em hospícios e o desenvolvimento de tratamentos alternativos que visem a reinclusão social final do doente.

Nesse sentido, demonstra ser importante a atenção conferida as doenças mentais desenvolvidas por crianças, tendo em vista ser um período da existência humana de desenvolvimento fisiológico e psicológico, em que o ser precisa de mais atenção e apoio. Assim, o estudo e desenvolvimento de métodos de cuidados com crianças e adolescentes enfermos mentais é de grande importância. 
Com isso, como analisaremos nesse estudo, para auxiliar o direcionamento e tratamento de enfermidades mentais o serviço de saúde de atenção primária deve estar qualificado e devidamente orientado para tratar esses tipos de caso, principalmente em razão da saúde pública ser utilizada pela maioria da população, bem como devido ao fato de ser de responsabilidade do Estado garantir a saúde de forma integral e universal, conforme determina a Carta Constitucional.

Isso revela a importância do presente estudo, tendo em vista que é preciso analisar os cuidados com saúde mental dispensados as crianças e adolescentes por uma perspectiva histórica, para fins de atestar a importância do desenvolvimento de políticas públicas específicas para os cuidados desse grupo etário, principalmente quanto a atenção primária.

\section{Breve histórico da saúde mental infantil no Brasil}

O estágio pueril, consiste período determinante na vida do ser humano, visto que se trata de momento de construção do seu físico e psicológico, bem como do desenvolvimento de habilidades e capacidades. Assim, todos os fatos ocorridos nesse ínterim são fundamentais e interferem diretamente na formação íntima e pessoal do indivíduo.

Apesar da importância desse momento da vida, a legislação de proteção aos menores apenas surgiu, no Brasil, no século XX. Esse arcabouço normativo tinha por objetivo educar o povo e, ainda, prevenir a desordem, já que oferece apoio às famílias que possuem dificuldade com seus filhos insubordinados. Para tanto, as crianças ou adolescentes poderiam ser entregues a tutela do Estado caso necessário, inclusive se a condição financeira precária impossibilitasse a família de conferir uma vida "digna" ao menor.

De maneira semelhante, foi tardia a importância dada ao campo de cuidado com a saúde mental das crianças no país, que ocorreu apenas no final do século XIX, quando surgiram os primeiros estudos psicológicos e psiquiátricos sobre o tema. Contudo, foi a partir de 1852, com o advento do Hospício Dom Pedro II, primeiro hospital psiquiátrico do Brasil, que os estudos sobre a temática foram intensificados. Sequencialmente, no início do século XX, houve o início de importante movimento, considerado marco na psiquiatria infantil brasileira, o qual foi 
denominado de movimento higienista, que consistiu em momento em que a medicina passou a demonstrar preocupação com essa patologia e sua interferência no desenvolvimento infantil.

O movimento higienista (Soares, 1990) ou sanitarista (Hochman, 1998) trouxe reinterpretações e novos ideais ao país, principalmente sobre os conteúdos relacionado com a área da saúde, em especial relacionados com a Saúde Pública, na Educação, e no estabelecimento e ensino de novos hábitos. Ou seja, era um movimento demasiadamente heterogêneo e ideológico, que buscou valorizar a população, tendo em vista que esta é o principal recurso da nação (Rabinbach, 1992). A homogeneidade do higienismo era encontrada no objetivo central de estabelecer novas normas e orientações com fulcro de aprimorar a saúde pública e individual, visto que a busca principal era desenvolver normas e hábitos que aprimorassem a saúde como um todo.

Com isso, junto ao higienismo, deu-se início no país a um desdobramento do movimento, qual seja, o Movimento de Higiene Mental. Esse movimento, estabelecido com o surgimento da Liga Brasileira de Higiene Mental, tem como objetivo analisar a saúde psíquica da população, e entendia acerca da necessidade de desenvolver uma atenção preventiva e coletiva com a população além da atividade curativa propriamente dita, que seriam direcionadas para diversos campos sociais, em especial para a família (Boarini, 2012).

Nessa época, as crianças "normais" participavam de escolas e internatos, enquanto aquelas que eram consideradas como portadoras de doenças mentais, eram internadas em hospícios, muitas vezes em conjunto com adultos enfermos, visto que não existiam estudos, tampouco instituições especificas para menores. Assim, os primeiros estudiosos da saúde mental infantil tentaram encontrar uma forma de remediá-la, entendendo que seria necessário investir a aperfeiçoar a assistência pública existente nesses manicômios. Conforme comentado, buscou-se investir na prevenção das enfermidades, além da cura efetiva, o que ressaltou a importância de um estudo aprofundado técnico-científico da psiquiatria infantil.

A sanidade mental infanto-juvenil, trata-se de fato nacionalmente reconhecido como importante, sendo enquadrado como questão de saúde pública, devendo, dessa forma, fazer parte das assistências disponibilizadas pelo Sistema Único de Saúde (SUS), que antigamente eram de responsabilidade da Educação e Assistência Social (Couto, Duarte \& Delgado 2008, p. 390-398). Hodiernamente, diversos são os números de especialidades que se preocupam com o desenvolvimento dos menores, envolvendo as áreas de neurologia, a psicologia, a psiquiatria, 
pedagogia, a fonoaudiologia, entre outras correlacionas com a assistência médica e patologizante das crianças e adolescentes.

Hodiernamente, conforme pode se inferir da Política Nacional de Saúde Mental, é preciso que as ações direcionadas para a saúde mental sejam desenvolvidas em conjunto com a atenção básica, em razão da similaridade de princípios. Dessa forma, o tratamento de saúde metal disponibilizado pela atenção básica do sistema público de saúde, deve realizar as ações de acolhimento e vínculo, a realização do trabalho em equipe e em rede, conferir os cuidados integrais, priorizar a desinstitucionalização, bem como buscar a reabilitação psicossocial da criança enferma, tendo em vista que esses consistem nos pontos de convergência entre essas políticas.

\section{Do direito à saúde pública}

Em 1940, juntamente a criação da Organização das Nações Unidas (ONU), surgiu o respeito nacional e internacional dos direitos do homem. Na mesma época, uma série de direitos, dentre os quais encontra-se o direito à saúde, foram enfatizados, tendo em vista sua importância, o que passou a demandar do estado maiores ações com escopo de assegurá-los por meio principalmente de políticas públicas (RODRIGUEZ, 2014).

Com isso, a Constituição da República Federativa do Brasil, definiu em seu artigo $6^{\circ}$ que "são direitos sociais a educação, a saúde, a alimentação, o trabalho, a moradia, o transporte, o lazer, a segurança, a previdência social, a proteção à maternidade e à infância, a assistência aos desamparados", colocando a saúde como direito fundamentalmente posto, sendo, além de condição para a efetiva dignidade da pessoa humana, dever Estatal garantir sua eficácia.

Nesse sentido, os serviços de saúde pública são entendidos como de relevância pública, não só estando submetidos ao controle social e do Estado, como também sendo de responsabilidade estatal oferecê-los, bem como impedir possíveis abusos a esse direito, conforme determina o artigo 197 da Carta Constitucional de 1988 que determina que "são de relevância pública as ações e serviços de saúde, cabendo ao poder público dispor, nos termos da lei, sobre sua regulamentação, fiscalização e controle, devendo sua execução ser feita diretamente ou através de terceiros (...)". No entanto, apesar da saúde ser direito de todos 
constitucionalmente estabelecido, este ainda não possui plena efetividade, o que é visível na precariedade do sistema público de saúde vigente.

O sistema público de saúde do Brasil está subdividido em: (i) Sistema Único de Saúde (SUS), que consiste em um dos maiores sistemas de saúde internacionalmente reconhecido, que tem o objetivo de fornecer um atendimento integral e universal à população; e (ii) Sistema de Saúde Suplementar, que conforme o próprio nome acusa, trata-se de sistema que busca complementar a saúde pública, e são disponibilizados pelos planos de saúde. Sendo assim, o setor de saúde do país detém de um modelo que utiliza de um sistema público (SUS), associado a um sistema privado (planos de saúde), que possuem diferentes objetivos, mas se complementam.

O SUS surge com o advento da Carta Magna de 1988, que elencou a saúde dentre os direitos considerados fundamentais para o indivíduo, bem como trouxe expresso no artigo 196 que "a saúde é direito de todos e dever do Estado, garantido por meio de políticas sociais e econômicas que visem à redução do risco de doença e de outros agravos e ao acesso universal e igualitário às ações e serviços para sua promoção, proteção e recuperação” (OLIVEIRA, 2010), revelando não só o dever do estado em conferir a integralidade da cobertura, como também sua universalidade.

Dessa forma, o sistema público de saúde brasileiro demonstra-se demasiadamente complexo e carece de efetividade em razão da ausência de políticas públicas suficientes para tanto. Contudo, sendo direito constitucionalmente posto, inclusive classificado como fundamental, deve o Estado unir mais esforços no sentido de possibilitar um sistema mais completo para a população.

\section{Da atenção primária disponibilizada pelo SUS às crianças com enfermidades mentais}

A Reforma Psiquiátrica no Brasil, iniciado nos anos 70, foi um evento sociopolítico que envolveu diversos atores sociais, instituições, territórios, mercados, dentre outros; que teve por objetivo a modificação e remodelação de práticas, conceitos e valores socioculturais relacionados com a temática. O movimento foi apoiado por diversos profissionais, como psicólogos, psiquiatras e outros profissionais da saúde, que reivindicaram a humanização dos hospitais psiquiátricos, com a criação de um local mais humanizado para os enfermos mentais, 
isto porque era cruel a forma como eram tratados como loucos, bem como a diminuição de leitos psiquiátricos e aumento dos serviços substitutivos de tratamento.

Os principais objetivos da reforma envolviam a criação de um ambiente mais humanizado para o tratamento dos doentes mentais e a exclusão de todos os manicômios, visto que não possuíam um tratamento eficaz, e, inclusive os pacientes por vezes eram internados e esquecidos pela família, o que gerava uma exclusão social do enfermo. Nesse sentido, o movimento possibilitou diversos avanços para a área, principalmente em relação ao tratamento conferido aos enfermos mentais.

Diversas conferências sobre o tema foram realizadas, em que se divulgava novos conceitos, táticas, métodos etc; bem como apontou-se a necessidade de redefinição de ações político-assistenciais de cuidado e tratamento da saúde mental infanto-juvenil, apresentada na IV Conferência Nacional de Saúde Mental Intersetorial de 2010.

O relatório da IV Conferencia indicou a necessidade de realização de ações intersetoriais e transdisciplinares, para fins de promover e assistir os enfermos mentais da forma mais completa possível, nos três níveis de atenção à saúde. Ademais, apontou que para a devida consolidação das políticas públicas voltadas para a saúde psíquica e psicológica das crianças e adolescentes, é preciso construir uma rede que abranja tanto os grandes quanto os pequenos centros populacionais. Com isso, inicia-se a remodelação da assistência à saúde mental infantojuvenil, que envolve pressupostos de inclusão social e diminuição do sofrimento dos enfermos internados, por meio da criação de uma rede alternativa de cuidados ao internamento em hospital psiquiátrico.

Hodiernamente, o sistema de saúde possui uma ampla rede de atenção aos enfermos mentais, inclusive infantis, detentora de serviços abertos e ações territoriais, que visam a diminuição dos leitos psiquiátricos, bem como a redução da exclusão social, com fulcro de acabar de uma vez por todas com os hospícios.

Contudo, apesar de tantas transformações, o movimento reformista ainda precisa enfrentar diversos desafios, como os relacionados à implementação das práticas voltadas para garantir os direitos básicos das crianças e adolescentes, dentre os quais se encontra o direito a ser assistido de forma integral pelo sistema público de saúde.

Hodiernamente, conforme Política Nacional de Saúde Mental, as ações envolvendo saúde mental devem estar articuladas a Atenção Básica, em razão da convergência principiológica. Assim, as ações devem envolver acolhimento, trabalho em equipe e em rede 
territorial, integralidade do cuidado, desinstitucionalização e reabilitação do paciente. (Borges, 2012). Logo, preciso investir em uma relação que torne harmônica as ações de saúde mental em conjunto com a atenção primária, para que seja possível cumprir as determinações da Reforma Sanitária e da Reforma Psiquiátrica no Brasil.

Para os pacientes com enfermidades severas e persistentes, implantou-se os Centros de Atenção Psicossocial (CAPS), que deixam em segundo plano os transtornos menos graves, o que, s em dúvidas, ainda carece de ajustes. Esses centros são serviços públicos de atenção intensiva, possuem como foco a desospitalização e desinstitucionalização, contudo não são suficientes para atender a demanda de enfermos que carecem de ajuda. Ademais, em regra, esses serviços não estão presentes nos pequenos municípios, que apenas detém de atenção primária e, por vezes, só possuem o ambulatório para esse fim, responsável por cuidar das crianças portadoras de doenças mentais.

Todas as medidas descritas, buscam diminuir os problemas da ausência de uma atenção primária qualificada para atender demandas de saúde mental, que ainda carece de ajustes. É o desenvolvimento da atenção primária aos enfermos mentais, tendo em vista que este é o primeiro contato do paciente com o sistema de saúde, sendo importante haver profissionais capazes de identificar a enfermidade para, assim, direcionar ou escolher a melhor medida a ser tomada a depender do caso, principalmente em se tratando de crianças, tendo em vista sua fragilidade.

Sendo assim, é notório que o campo da saúde, consiste em área importante para os cuidados mentais das crianças. Em especial a atenção básica, o campo educacional e a seara assistencial, merecem atenção, tendo em vista que são pontos estratégicos no desenvolvimento da saúde mental infantil, já que são os campos de maior contato das crianças com o mundo.

Logo, uma rede pública de saúde precisa dispor de uma atenção primária direcionada aos cuidados com enfermidades mentais da população infanto-juvenil, devendo estar preparada para os diferentes graus de complexibilidade, sabendo a melhor forma de intervir, devendo, dessa forma, possuir uma estrutura capaz de identificar e resolver as diversas enfermidades mentais que uma criança pode apresentar. (COUTO et al, 2008, p. 390-398). Portanto, resta preciso o investimento em profissionais qualificados na atenção primária de saúde, para que seja possível a entrega de uma ação de cuidado integral de saúde. 


\section{Considerações Finais}

Conforme comentado, a maioria dos estudos, políticas e serviços assistenciais relacionados à saúde mental, são direcionados aos adultos. Contudo, os transtornos infantojuvenis possuem particularidades de risco e proteção, que carecem de estratégias específicas, não sendo suficiente a adaptação do modelo adulto para tratá-los.

Sem dúvidas, o estudo revela a existência de três principais problemas a serem enfrentados, sendo eles: (i) a expansão dos serviços de saúde, especificamente dos CAPSi e dos ambulatórios de saúde mental infantil; (ii) a gestão territorial, que constitui um entrave, visto que o tratamento de uma enfermidade mental carece de acompanhamento constante; e (iii) possibilidade dos menores serem atendidos em CAPS e ambulatórios não específicos, se necessário, sendo necessário que o corpo profissional seja devidamente qualificado para tanto.

Sendo assim, o Estado ainda é incapaz de fornecer a saúde pública com integralidade para a população. Ainda, a ausência de diretrizes políticas no sentido de elaborar e implementar ações assecuratórias da saúde mental infanto-juvenil, significa um sério problema, que tem tentado ser remediado por instituições privadas ou filantrópicas, o que, infelizmente, tem gerado situações de desassistência, abandono e exclusão.

Isso demonstra que, apesar dos avanços trazidos pela Reforma Psiquiátrica, o país ainda precisa enfrentar diversos desafios, dentre eles o de desenvolver políticas públicas capazes de assegurar os direitos fundamentais básicos da população, inclusive das crianças. Isso é notório principalmente na ausência de atenção primária para cuidar dos problemas mentais das crianças, se necessário, constituindo, dessa forma um paradigma da atenção psicossocial.

É preciso a edição de políticas e ações específicas para o cuidado da população infantil, que apena possuíam modelos adaptados para eles, sem abranger suas especificidades; ademais, é imperioso a disponibilização dos cuidados coma saúde mental infantil na atenção primária, juntamente com qualificação dos profissionais para atuarem nesse setor. Sendo assim, a construção de políticas voltadas para as crianças e adolescentes, trata-se de um grande desafio para o campo da saúde mental, visto que é preciso conciliar as particularidades desse grupo com os princípios estabelecidos pelo SUS.

Dessa forma, a carência de políticas de saúde mental específicas direcionadas ao público infantil acusam a urgência do desenvolvimento dessas, sendo necessário o desenvolvimento de estudos para fins de arrecadar dados e informações culturalmente relevantes, para que seja 
possível elaborar políticas adequadas, principalmente em relação a atenção primária, em razão de ser etapa fundamental no sistema de saúde.

\section{Referências}

AGÊNCIA NACIONAL DE SAÚDE SUPLEMENTAR. Caderno de Informação de Saúde Suplementar: beneficiários, operadoras e planos. Rio de Janeiro: ANS, junho de 2013. Disponível em http://www.ans.gov.br/images/stories/Materiais_para_pesquisa/Perfil_setor/Caderno_informa cao_saude_suplementar/2013_mes12_caderno_informacao.pdf > . Acesso em 23/01/2018.

AGÊNCIA NACIONAL DE SAÚDE SUPLEMENTAR. Caderno de Informação de Saúde Suplementar: beneficiários, operadoras e planos. Rio de Janeiro: ANS, junho de 2016. Disponível em: < http://www.ans.gov.br/images/stories/Materiais_para_pesquisa/Perfil_setor/Caderno_informa cao_saude_suplementar/caderno_JUNHO_2016_total.pdf>. Acesso em: 23/01/2018.

BASTOS, H. P. (2013). Saúde e Educação: reflexões sobre o processo de medicalização. Tese de Doutorado, IPUSP, USP - São Paulo, SP, Brasil.

BOARINI, M. L. (Org.). (2012). Higiene Mental: Ideias que atravessaram o século XX. Maringá: EDUEM.

BORGES, R.F. (2012). Reforma Psiquiátrica Brasileira e Atenção Básica à Saúde: desafios e possibilidades. Tese de Doutorado. UNESP, Assis, SP, Brasil. 2012. Disponível em <https://repositorio.unesp.br/handle/11449/105614>. Acesso em: 08/07/2018.

COUTO, M. C. V.; DUARTE, C. S.; DELGADO, P. G. G. A saúde mental infantil na Saúde Pública brasileira: situação atual e desafios. Revista Brasileira de Psiquiatria. 2008;30(4):3908. Disponível em: 〈http://www.scielo.br/pdf/rbp/v30n4/a15v30n4>. Acesso em 08/07/2018.

DALLORA, M. E. L. V. Gerenciamento de custos de matérias de consumo de um hospital de ensino. Ribeirão $2007 . \quad$ preto, Disponível $\quad$ em <http://www.teses.usp.br/teses/disponiveis/17/17139/tde-03032008-133139/pt-br.php>. Acesso em: 08/07/2018.

FAVERET, P., OLIVEIRA, P.J. A universalização excludente: reflexões sobre as tendências do sistema de saúde. Planejamento e Políticas Públicas, nº 3, p. 139-162, IPEA, 1990.

MARQUES FILHO, J. A Dimensão bioética dos conflitos de interesses na relação entre médico e indústria farmacêutica. Rev. Bras. Clin. Med. V. 8, n. 2, 2010. Disponível em: <http://files.bvs.br/upload/S/1679-1010/2010/v8n2/a011.pdf>. Acesso em 08/07/2018. 
OLIVEIRA, J. A. D. Demandas jurídicas por coberturas assistenciais - estudo de caso; CASSI. 2010. Dissertação (Mestrado em Saúde Pública) - Faculdade de Saúde Pública, Universidade de São Paulo, São Paulo.

GÓIS JÚNIOR, E. Movimento higienista e o processo civilizador: apontamentos metodológicos. Disponível em: <http://www.uel.br/grupoestudo/processoscivilizadores/portugues/sitesanais/anais10/Artigos_PDF/Edivaldo_Gois_Jr.p df>. Acesso em 08/07/2018.

HOCHMAN, Gilberto; LIMA, Nízia. Condenado pela raça, absolvido pela medicina: o Brasil descoberto pelo Movimento Sanitarista da Primeira república. In: Raça, ciência e sociedade. Rio de Janeiro, Fiocruz, 1996.

MAXIMILIANO, Carlos. Hermenêutica e Aplicação do Direito. 19 ed. Rio de Janeiro: Forense, 2010.

ILICH, I. (1975). A expropriação da saúde: nêmeses da medicina. Rio de Janeiro: Editora Nova Fronteira.

LUZIO, C.A. (2003). A Atenção em Saúde Mental em Municípios de pequeno e médio portes: Ressonâncias da Reforma Psiquiátrica. Tese de Doutorado em Saúde Coletiva - Faculdade de Ciências Medicas, Universidade de Campinas, Campinas, SP, Brasil.

MENDES, E.V. (2001). Os grandes dilemas do SUS: tomo II. Salvador, BA: Casa da Qualidade editora. Postman, N. (1999). O Desaparecimento da infância. Rio de Janeiro Graphia,1999.

MINISTÉRIO DA SAÚDE. Reforma Psiquiátrica e política de Saúde Mental no Brasil. Conferência Regional de Reforma dos Serviços de Saúde Mental: 15 anos depois de Caracas. Disponível em: <http://bvsms.saude.gov.br/bvs/publicacoes/Relatorio15_anos_Caracas.pdf>. Acesso: 08/07/2018.

RIBEIRO, P.R.M. (2006). História da Saúde Mental Infantil: A Criança Brasileira da Colônia à República Velha. Psicologia em Estudo, Maringá, v. 11, n. 1, p. 29-38.

RIZZINI, I. (2008). O século perdido: raízes históricas das políticas públicas para a infância no Brasil. São Paulo: Cortez.

RODRIGUEZ, A. M. M. M. O processo da judicialização da saúde como via de acesso à atenção integral: o retratoissertação de um município de grande porte do estado de São Paulo. 2014. 152f. Dissertação (Mestrado) - Escola de Enfermagem de Ribeirão Preto, Universidade de São Paulo, Ribeirão Preto, 2014.

SCHEFFER, M. Os planos de saúde nos Tribunais: uma análise das ações judiciais movidas por clientes de planos de saúde, relacionadas a negativas de coberturas assistenciais no estado de São Paulo. 2006. Dissertação (Mestrado) - Faculdade de Medicina, Universidade de São Paulo, Ribeirão Preto, 2006. 
SISTEMA ÚNICO DE SAÚDE. Conselho Nacional de Saúde. (2010). Comissão Organizadora da IV Conferência Nacional de Saúde Mental - Intersetorial. Relatório Final da IV Conferência Nacional de Saúde Mental - Intersetorial, 27 de junho a 1 de julho de 2010. Brasília: Conselho Nacional de Saúde/Ministério da Saúde.

SOARES, Carmem. O pensamento médico higienista e a Educação Física no Brasil: (18501930). São Paulo, 1990. Dissertação (Mestrado) - Pontifícia Universidade Católica de São Paulo.

\section{Como citar este artigo (Formato ABNT):}

OLIVEIRA, Sidney Medeiros de; SILVA, Messias Santos da; NOGUEIRA, Michelle Carolina Alves. Saúde mental infanto-juvenil: a necessidade de políticas públicas mais efetivas pelo Estado. Id on Line Rev.Mult. Psic., 2018, vol.12, n.41, p.748-759. ISSN: 1981-1179.

Recebido: 04/07/2018

Aceito 13/07/2018 